\section{INDICADORES DE GOBERNABILIDAD Y POSCONFLICTO EN EL MUNICIPIO DE VALLEDUPAR, COLOMBIA}

\author{
INDICATORS OF GOVERNABILITY AND POSTCONFLICT \\ IN THE MUNICIPALITY OF VALLEDUPAR, COLOMBIA
}

\section{Antonio Yesid Pedroza Estrada}

\section{RESUMEN}

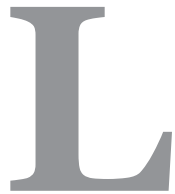

a gobernabilidad, es vista como el arte de gobernar bien o el ejercicio del buen gobierno para producir los resultados que la sociedad espera de sus gobernantes. Esta investigación evalúa el nivel de logro de los indicadores de gobernabilidad, de acuerdo con el modelo aplicado por el Instituto del Banco Mundial (IBM, 2017), en el municipio de Valledupar, Colombia. Se aplicó un diseño cuantitativo, el tipo de investigación es descriptiva y propositiva, se proponen nuevas formas de aplicación del conocimiento a la realidad encontrada en el ámbito local para mejorar las condiciones de vida de la ciudadanía. El instrumento aplicado fue estructurado con base en los seis indicadores propuestos por el IBM, y se agregó otro relacionado con la actitud de la administración local, para adaptar la gobernabilidad al nuevo escenario producto de los acuerdos entre el gobierno colombiano y las Fuerzas Armadas Revolucionaria Colombiana (FARC). Se sistematizó, procesó, analizó e interpretó la información obtenida por los investigadores, y se propone, a la Administración Municipal, una serie de instrumentos, estrategias y dinámicas que ayudaran a mejorar los índices de gobernabilidad y apoyo social a las políticas públicas municipales que coadyuven en fortalecer un escenario constructivo de paz en el posconflicto.

Palabras clave: buen gobierno, administración municipal, posconflicto.

\section{ABSTRACT}

Governance is seen as the art of governing well or the exercise of good governance to produce the results that society expects from its rulers. This research evaluates the level of achievement of the governance indicators, in accordance with the model applied by the World Bank Institute(IBM, 2017), in the municipality of Valledupar, Colombia. A quantitative design was applied, the type of research is descriptive and proactive, new forms of application of
Antonio Yesid Pedroza Estrada yesithpedroza@hotmail.com

UPC Escuela Superior de Administración Pública, ESAP

Universidad Popular del Cesar COLOMBIA

COMO CITAR ESTE ARTÍCULO Pedroza Estrada, A. Y. (2019). Indicadores de gobernabilidad y posconflicto en el Municipio de gobernabilidad y posconflicto en el Municipio de Valledupar, Colombia. Revista de la Facultad de Ciencias Económicas, 23(2), 129 - 153. http://dx.doi.org/10.30972/rfce.2324023

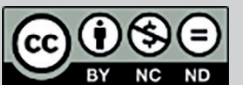
https://creativecommons.org/licenses/by-nc-nd/4.0/ Revista de la Facultad de Ciencias Económicas ISSN 1668-6357 (formato impreso) ISSN 1668-6365 (formato digital) por Facultad de Ciencias Económicas Universidad Nacional del Nordeste (UNNE) Argentina se distribuye bajo una Licencia Creative Commons Atribución - No Comercial - Sin Obra Derivada 4.o Internacional. 
knowledge are proposed to the reality found at the local level to improve the living conditions of the citizens. The applied instrument was structured based on the six indicators proposed by the IBM, and another one related to the attitude of the local administration was added, to adapt the governability to the new scenario resulting from the agreements between the Colombian government and the Revolutionary Colombian Armed Forces. (FARC). The information obtained by the researchers was systematized, processed, analyzed and interpreted, and a series of instruments, strategies and dynamics are proposed to the Municipal Administration that will help improve the governance and social support indexes of the municipal public policies that contribute in strengthening a constructive peace scenario in the post-conflict.

Keywords: good government, municipal administration, post-conflict.

\section{INTRODUCCIÓN}

La administración de lo público, se percibe por la sociedad con un carácter problemático y complejo, es decir, los problemas de gobernabilidad que las autoridades públicas deben enfrentar cotidianamente, en desarrollo de la vida social y política de las comunidades, con variables inmanejables como: ineficiencia, corrupción, pobreza, miseria y hambre; situación que conlleva a la pérdida de credibilidad en las instituciones públicas, desencanto y alejamiento del ciudadano de la actividad proselitista como opción política.

La gobernabilidad, vista como el arte de gobernar bien o el ejercicio del buen gobierno, para producir los resultados que la sociedad espera de sus gobernantes, es el deber ser, lo que se espera que realice un gobierno, sea cual sea su espacio. Sin embargo, en el ejercicio real de administración pública incide una variable que determina la forma estructural de llevarlo a cabo, es el ser humano; quien es sujeto de toda la dinámica de contradicciones, sentimientos, ideología, intereses, etc. que lo forman. La gestión pública es compleja porque así lo es el ser humano.

Para intentar disminuir la anterior complejidad, se han inventado procesos, procedimientos, normas, fórmulas, que procuran enmarcar dentro de un contexto científico, y que buscan propiciar que el ejercicio de gobierno sea objetivo, de tal manera, que la administración pública sea trasparente, eficaz, eficiente, legal, entre las tantas características que debe tener un buen gobierno. Para el ejercicio de la gobernabilidad se ha trabajado por la implementación de unos criterios denominados índices de gobernabilidad, como especie de faro o semáforo que indique la forma como se desarrollan los objetivos de gobierno enfrentados a las necesidades y sentir de las comunidades. Eso es lo que se pretende en esta investigación, auscultar 
como percibe la sociedad del municipio de Valledupar la ejecución del actual gobernante local, mediante la utilización de un instrumento de medición enmarcado en criterios científicos.

La situación esbozada anteriormente, induce al planteamiento de interrogantes que se pretenden responder en este artículo tomando criterios de instituciones internacionales que han tenido éxito realizando estudios de gobernabilidad a nivel nacional y territorial en Colombia y otros países como el Instituto del Banco Mundial, el Programa de Naciones Unidas para el Desarrollo, PNUD, a través del Centro de Gobernabilidad de Oslo (2017) y el Club de Roma (1996):

¿Cuál es el nivel de logro de los indicadores de gobernabilidad, de acuerdo con el modelo aplicado por el Instituto del Banco Mundial (IBM), que actualmente muestra la administración del municipio de Valledupar, Colombia?

¿Cuáles son los indicadores de gobernabilidad más efectivos para mostrar la gestión del municipio de Valledupar de acuerdo con el modelo aplicado por el Instituto del Banco Mundial (IBM)?

¿Qué tipo de proceso investigativo debe aplicarse para el estudio de los indicadores de gobernabilidad, del municipio de Valledupar, con relación a las teorías de instituciones internacionales como Instituto del Banco Mundial (IBM)?

¿Cuáles instrumentos de carácter práctico se deben utilizar para monitorear y controlar el nivel de desempeño de los indicadores de gobernabilidad en la gestión pública municipal para fortalecer la democracia en la fase de posconflicto nacional en Colombia?

\section{OBJETIVOS}

\section{Objetivo General}

Evaluar el nivel de logro de los indicadores de gobernabilidad, de acuerdo con el modelo aplicado por el Instituto del Banco Mundial (IBM), que muestra la administración del municipio de Valledupar, Colombia.

\section{Objetivos Específicos}

Identificar los indicadores de gobernabilidad de la gestión del municipio de Valledupar de acuerdo con el modelo aplicado por el Instituto del Banco Mundial (IBM).

Analizar los indicadores de gobernabilidad, del municipio de Valledupar, producto de la aplicación de un instrumento de medición cuantitativo, con relación a las teorías de instituciones internacionales.

Proponer la implementación de instrumentos de carácter práctico para monitorear y controlar el nivel de desempeño de los indicadores de gobernabilidad en la gestión pública municipal para fortalecer la democracia en la fase de posconflicto nacional. 


\section{METODOLOGÍA}

La investigación se centró en los indicadores de gobernabilidad, temática enlazada con dos líneas institucionales de investigación de la Escuela Superior de Administración Pública (ESAP): Ciudadanía y construcción de lo público (sublínea d. Gobernabilidad y administración pública) y Políticas públicas y gobernabilidad (sublínea e. Políticas de fortalecimiento cívico-ciudadano).

Metodológicamente, se aplicó un diseño cuantitativo, el tipo de investigación es descriptiva, ya que describe la opinión de un grupo de ciudadanos sobre un tema de relevancia municipal, tal como lo plantea Hernández, Fernández y Baptista (2016) y propositiva, ya que el mismo se proponen nuevas formas de aplicación del conocimiento (Hurtado, 2010).

Para el diseño del cuestionario, como instrumento, se hicieron preguntas que mostrarán la percepción a nivel local de un grupo representativo del municipio de Valledupar. El cuestionario se aplicó personalmente por los investigadores y por los estudiantes pertenecientes a los dos (2) semilleros adscritos a la investigación, en su desarrollo, se le hacía una breve introducción a los encuestados sobre el objetivo de la misma, la reserva de la información, luego se le entregaba junto con lapicero, y se le pedía que respondieran, personalmente, según su criterio, sin insinuaciones o direccionamiento.

Respecto al cálculo del tamaño de la muestra se utilizó el método probabilístico, tomando como N, es decir, el tamaño de la población, la del municipio de Valledupar, igual a 413.341 habitantes, información tomada de la página oficial del Municipio de Valledupar (2017) con un error estándar del $5 \%$, por ciento, o sea, con un nivel de confianza del 95\%, arrojando como resultado la cantidad de trescientos ochenta y cuatro (384) muestras, las que se hicieron en un lapso de dos (2) semanas, por intermedio de diez (10) personas: dos (2) investigadores y ocho (8) estudiantes de los dos semilleros adscritos a la investigación.

En cuanto al tratamiento de la información se diseñó un aplicativo en Microsoft Access, diseñado por uno de los estudiantes perteneciente a uno de los semilleros adscritos, el cual le fue entregado a cada una de las personas que realizaron las encuestas para que digitaran los resultados. Una vez obtenida la información final, se verificó su digitación, procurando disminuir los errores, y se procedió a la realización de información estadística con gráficas para que fueran interpretadas por los investigadores. Finalmente se realiza un análisis holístico con relación al marco contextual de la investigación, de donde fluyeron inferencias y conclusiones cuyos resultados se sintetizan en el presente artículo.

Del estudio de los resultados se logró información que muestra el nivel de percepción que la ciudadanía y los actores sociales, tienen de la eficacia de los indicadores de gobernabilidad y de la forma como son gobernados en el municipio de Valledupar, incluyendo reconocimientos de logros y falencias. Basados en los resultados y en teorías vigentes, se propondrá un instrumento práctico que permita a los gobernantes locales revisar permanentemente el 
avance de su gestión, a través de los indicadores de gobernabilidad pertinentes a su territorio, el cual, dado su carácter estandarizado, podrá ser aplicado en cualquier municipio.

\section{CONTEXTO}

\section{SOBRE LA GOBERNABILIDAD}

Investigadores del Banco Mundial y del Brooking Institut, definen gobernabilidad como: "las tradiciones e instituciones a través de las cuales se ejerce la autoridad, es la capacidad para formular y aplicar eficazmente políticas acertadas y el respeto a la ciudadanía” (Hernández, 2016, p.6).

En este sentido, una deficiente gobernabilidad provoca la involución de amplios sectores que se desorganizan y retroceden a estados de ingobernabilidad, a la incivilidad y la barbarie; y arrastran en su retroceso a la sociedad en su conjunto, que privada de la confianza en las leyes y los gobernantes pierden el sentido del orden y el rumbo del desarrollo. (Hernández, 2016, p.4).

Por otro lado, el concepto de liderazgo normalmente está compuesto de un proceso que involucra una relación de influencia en doble sentido, orientado especialmente a la atención de objetivos mutuos, como aquel de un grupo, institución o sociedad. Así, el liderazgo no requiere solamente el cargo del líder, sino también requiere esfuerzo de cooperación de parte de las otras personas (Hollander, 1978). Percibimos que el tema está cargado de complejidad, así como la propia universidad, pues hay personas involucradas y el ser humano, de una forma u otra, trae consigo percepciones y vivencias distintas (Fossatti, 2013).

Con relación a los rasgos y estilos de liderazgo, Bryman (1992) explica que los conceptos teóricos relacionados con los rasgos de personalidad son más antiguos con respecto al liderazgo. Uno de ellos es una característica distinta de la personalidad. Esas teorías identifican al líder como aquel que posee rasgos específicos de personalidad que lo difieren de las demás personas. Así, el líder presenta características de personalidad por medio de las cuales podrá influir en el comportamiento de los demás. Como premisa de esta teoría, los líderes poseen rasgos de personalidad por los cuales el individuo ya nace líder. Bryman (1992) asimismo señala tres importantes aspectos con relación a los rasgos sociales, aquellos relacionados con la tarea, desde la perspectiva del comportamiento de los seguidores: a) la influencia que el líder ejerce sobre los demás, el que de alguna manera induce su comportamiento; b) la concepto de este proceso de influencia como incorporado al contexto de un grupo; c) la influencia ejercida por el líder sobre el comportamiento de los miembros de un grupo en dirección a los objetivos que pretenden alcanzar. Este conjunto conceptual lo va perfeccionando el mismo autor Bryman (1992), incluyendo asimismo las cuestiones culturales y de identificación - con la institución y con el grupo en sí mismo -. 
En el caso del presente proyecto de investigación se pretende medir los indicadores de gobernabilidad en el municipio de Valledupar pero con el objetivo de indicar la importancia de los mismos en desarrollo de la etapa de posconflicto en Colombia, con motivo de los acuerdos celebrados con las Fuerzas Armadas Revolucionaria de Colombia FARC, y las que se están desarrollando con el Ejército de Liberación Nacional ELN.

El posconflicto surge posteriormente a la cesación del enfrentamiento armado en forma definitiva o parcial, entre las fuerzas del Estado y otras, subversivas, que pretenden destruirlo o cambiarlo. Se espera que a partir de este momento entre a operar cambios en la sociedad sometida al conflicto armado. Nuevas instituciones gubernamentales, nuevas leyes, cambios constitucionales, innovaciones en la ejecución de gastos públicos, seguridad, entre otros, así como cambios estructúrales en la sociedad con respecto a los ámbitos políticos, sociales, educativos, económicos y algunos casos, hasta culturales. El fin último, es trabajar por la consolidación de una paz estable y duradera en Colombia, por lo que se espera el cumplimiento de los acuerdos suscritos entre los actores rebeldes y los estatales.

Para la Universidad del Rosario (2017) se debe estructurar bases sólidas para el fortalecimiento de la Paz, por lo que es vital la consolidación de importantes atributos en el posconflicto como son la reconciliación, la atención a población vulnerable, la construcción de memoria y verdad, la justicia transicional y la reparación, la prevención de la violencia y el crimen, la reforma de las Fuerzas Armadas y de Policía, la reconstrucción y el desarrollo económico, la estabilización política y la participación del sector privado, la sociedad civil y la comunidad internacional en todos ellos.

En la medida en que el Estado y los entes territoriales como los departamentos y los municipios colaboren con los objetivos y estrategias propuestas por el gobierno nacional, será más fácil la consolidación de la Paz; en este contexto, sin lugar a dudas, los indicadores de gobernabilidad se constituyen en una brújula que indica la dirección y la forma como se van alcanzando los cambios necesarios dentro de las instituciones públicas y de la sociedad colombiana en general.

\section{INDICADORES DE GOBERNABILIDAD}

El ejercicio de la gobernabilidad en cualquier ámbito espacial del territorio, debe ser eva-luado frecuentemente para que el gobernante disponga de elementos de juicio que le permitan redireccionar acciones y avanzar con firmeza en el proceso de construcción y consolidación del tejido social entre sus gobernados. Los indicadores de gobernabilidad son referentes reales y objetivos que conciernen a aspectos puntuales de las relaciones entre gobernantes y gobernados, y en gran medida su evaluación refleja el éxito o fracaso de una 
gestión; su valoración constante le dice al gobernante cuáles son sus niveles de logro y de legitimidad de la gestión adelantada, la cual debe ser evaluada constantemente sobre la marcha misma.

Para realizar esta medición, existen ya varias metodologías o más bien taxonomías y clasificaciones con las cuales se diseñan los instrumentos que sirven de base para realizar ejercicios de valoración de la gobernabilidad. Entre las organizaciones que han prestado especial interés a este asunto se encuentran el Banco Mundial (BM, 2017) con el Programa de Naciones Unidas para el Desarrollo, PNUD, a través del Centro de Gobernabilidad de Oslo (2017) y el Club de Roma (1996).

Con base en las propuestas y clasificaciones taxonómicas de estos organismos mundiales se han identificado seis ejes o núcleo moleculares, en torno a los cuales se agrupan categorías de variables genéricas, que sirven para diseñar los instrumentos para hacer las mediciones que fueron aplicados en desarrollo del proyecto.

\section{DISCUSIÓN E INFERENCIAS TEÓRICAS}

Cumplir con el objetivo general de la investigación, implica asumir el desarrollo de la gobernabilidad a través de la democracia como aspecto general, en un juego interrelacional de los actores que componen el cuerpo social, gobernantes y gobernados, lo cual impone la necesidad de fortalecer otras realidades más allá del simple concepto, y cuya dinamización es necesaria para su desarrollo.

El Centro de Gobernabilidad de Oslo define la gobernabilidad como "El resultado de interacciones, relaciones y redes entre los distintos sectores (instituciones de gobierno, sector público, sector privado y sociedad civil) e implica decisiones, negociación y distintas relaciones de poder entre las partes interesadas para determinar quién, cuándo y cómo se obtiene qué" (PNUD, s.f.: 13). De ahí que al definir la gobernabilidad el PNUD sostenga que:

Es el sistema de valores, políticas e instituciones por medio de las cuales una sociedad administra sus asuntos económicos, políticos y sociales a través de interacciones dentro de y entre el Estado, la sociedad civil y el sector privado. Es el modo en que una sociedad se organiza para tomar e implementar decisiones logrando un entendimiento, acuerdo y acción mutuos... Es el conjunto de reglas, instituciones y prácticas que fijan límites y proporcionan incentivos a los individuos, organizaciones y empresas. La gobernabilidad... opera en todos los niveles de la empresa humana, ya se trate del hogar, la aldea, la municipalidad, la nación, la región o el globo (p. 13, 2ed. S.f.). 
Según el Banco Mundial (BM), "La gobernabilidad de un país está asociada con la capacidad de sus instituciones para ejercer la autoridad pública e impulsar un desarrollo económico, social y político duradero" (2017:1).

El gobierno municipal es el foco de gestión institucional de gobernabilidad local. Pero la amplia esfera de gobernabilidad abarca un conjunto de instituciones estatales y no estatales, mecanismos y procesos mediante los cuales se prestan bienes y servicios públicos a los ciudadanos, con los cuales estos pueden articular sus intereses y necesidades, dirimir diferencias, ejercer derechos y obligaciones (PNUD, s.f.,:14).

Pero todo gobernante, cualquiera que sea el nivel de su jurisdicción (local, territorial regional o nacional), debe manejar criterios y elementos de juicio, para valorar el nivel de desenvolvimiento de su desempeño gubernativo, teniendo en cuenta que hay diferentes maneras de evaluarlo.

El gran dilema consiste en definir si se puede medir la gobernabilidad, es decir, la relación del gobierno y sus gobernados y cómo hacerlo; de ahí los frecuentes interrogantes acerca de ¿qué herramientas están a nuestro alcance para evaluar la gobernabilidad? ¿Qué son los indicadores de gobernabilidad? ¿La gobernabilidad es medible solo estadísticamente o se pueden emplear criterios cualitativos, para tener una idea clara de cómo va la gestión gubernativa en el territorio?

La gobernabilidad si es medible y desde hace un par de décadas han proliferado diversos tipos de técnicas de medición. Según Daniel Kauffman (2008) director de gobernabilidad del Instituto del Banco Mundial (BM), esto enfrenta al escepticismo, ya que los nuevos modelos y enfoques se concentran en crear parámetros e indicadores basados en instrumentos elaborados por expertos.

Organizaciones multilaterales como el BM, La ONU, La OEA y la Unión Europea (EU), han venido fomentando en las dos últimas décadas la creación de instituciones que convocan expertos que además de realizar estudios, con base en encuestas y sondeos que contienen datos sobre diferentes dimensiones de la gestión de los asuntos públicos, ha permitido crear un banco de datos sobre la gobernabilidad a nivel mundial (Kaufmann, 2008).

Frente a este panorama y teniendo en cuenta que el Gobierno Central de Colombia ha firmado con el grupo insurgente de las Farc el "Acuerdo final para la terminación del conflicto y la construcción de una paz estable y duradera”, se entiende que la paz del país no puede ser producto solo de un acuerdo y que si bien, parte de los problemas que en el pasado originaron el conflicto tienen sus raíces en las deficiencias de la gobernabilidad, hoy es necesario adecuar las condiciones de esa gobernabilidad para que desde lo local municipal, la sociedad civil y sus gobernantes vayan generando los aprestamientos exigidos para que esa paz, en realidad sea estable y duradera, lo cual será materialmente imposible sin una firme participación y el aporte decidido de la sociedad civil.

En ese sentido el proyecto se enfoca a evaluar las condiciones de gobernabilidad de un municipio en concreto, como es el de Valledupar, tercero más extenso de la región Caribe, con unas particularidades paradigmáticas como escenario en donde se configuraron particulares expresiones del conflicto armado vivido en el país y a partir de los resultados, se propondrá un 
modelo que permita a cada municipio del país evaluar su gobernabilidad local y adecuar sus condiciones para enfrentarse de manera proactiva al nuevo escenario de postconflicto que se avecina en el país, pues este tipo de mediciones pueden mejorar la comprensión de los déficits y flaquezas de la gobernabilidad y su relación con los impactos de desarrollo.

Estas evaluaciones deben proporcionar información relevante sobre asuntos específicos del nivel local, como las políticas relacionadas con la descentralización, rendición local de cuentas; respeto a los derechos humanos, justicia y equidad, ética pública, transparencia y participación ciudadana incluyente; y ser más sensibles a las necesidades particulares de los colectivos de partes interesadas y segmentos de la comunidad local.

\section{RESULTADOS}

Con la aplicación del instrumento, estructurado con base en los seis indicadores propuestos por el IBM, y de su sistematización se logró medir la percepción ciudadana sobre el desempeño de o comportamiento de los indicadores de gobernabilidad en el municipio de Valledupar.

A los seis indicadores, que fueron especialmente adaptados para los efectos de un estudio local, el grupo de investigación le agregó uno relacionado con la disponibilidad o actitud de la administración local, para adaptar la gobernabilidad al escenario de paz, teniendo en cuenta que en su entorno, a una hora, uno y a treinta minutos, otro, se encuentran localizadas dos zonas veredales, de las que fueron acordadas entre el gobierno y las FARC, para ambientar el proceso de retorno de los excombatientes a la vida ciudadana institucional; realidad que no puede ser ajena a la administración local, si se tiene en cuenta que esta población tiene en la ciudad el centro urbano más cercano y al cual mirarán como potencial centro para rehacer su proyecto de vida.

\section{ANÁLISIS DE RESULTADOS}

\subsection{ESTRUCTURA DEL INSTRUMENTO UTILIZADO}

El instrumento diseñado fue un cuestionario tipo encuesta con preguntas cerradas, propio de las investigaciones cuantitativas. La primera hoja de la encuesta presenta una información general de la institución y del grupo que desarrolla la investigación, seguidamente se le informa de que se trata el estudio y se le piden unos datos estadísticos que permitirán identificar los sujetos de estudio, según su género, edad, sector social y nivel de estudio, también, hay un aparte específico, en caso que el sujeto entrevistado sea funcionario público. 
Las siguientes dos páginas contienen en forma de tabla con columnas y filas, letras grandes para facilitar la lectura de los sujetos encuestados, desarrolladas las siete categorías, son las siguientes:

a) Categoría 1: Voces y Rendición de Cuentas

b) Categoría 2: Estabilidad Política y Ausencia de Violencia (percepción)

c) Categoría 3: Eficacia de la Acción Gubernativa (ejercicio de cumplimiento municipal)

d) Categoría 4: Calidad Regulatoria (fomento de normas regulatorias municipales)

e) Categoría 5: Imperio de la Ley (reflejo del respeto de la ley en el municipio)

f) Categoría 6: Control y Corrupción (control de la autoridad municipal)

g) Categoría 7: Gobernabilidad y Proceso de Paz (acciones del gobierno local)

Para la calificación de cada uno de los tópicos de las diferentes categorías según la opinión de los encuestados, se utilizaron intervalos con su respectiva cuantificación:

Tabla 1. Calificación tópicos según categorías

\begin{tabular}{|l|l|}
\hline \multicolumn{1}{|c|}{ Ítem } & \multicolumn{1}{c|}{ Valor } \\
\hline En total desacuerdo & 1 \\
\hline En desacuerdo & 2 \\
\hline No opina & 3 \\
\hline De acuerdo & 4 \\
\hline Totalmente de acuerdo & 5 \\
\hline
\end{tabular}

Fuente: Pedroza E. al.(2017)

\subsection{GRAFICACIÓN E INTERPRETACIÓN}

Los resultados de la aplicación de las encuestas, después de ser sistematizadas, arrojaron los siguientes resultados para los índices de gobernabilidad en el municipio de Valledupar:

Categoría 1: voces y rendición de cuentas (gobierno local estimula y respeta) Ítem 1. La cultura de la participación ciudadana

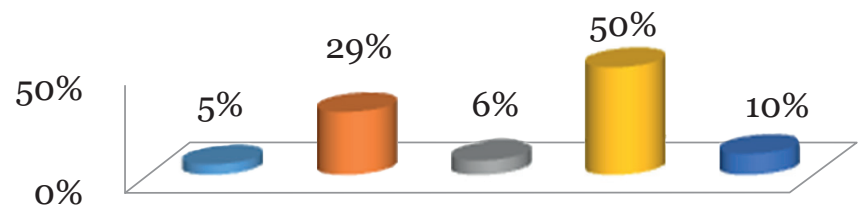

Más de la mitad de los encuestados, un 60\%, opina que el municipio de Valledupar está trabajando por la cultura ciudadana, perciben a través de las acciones del gobierno municipal que se estimula la participación ciudadana. Solamente el 34\% no está de acuerdo con lo preguntado, resaltándose que son la mitad de los que apoyan las acciones municipales. 
Ítem 2. La diversidad y libertad en la opinión pública y crítica ciudadana a sus acciones

EN EJERCICIO DE SU AUTORIDAD EL GOBIERNO LOCAL ESTIMULA Y RESPETA: La diversidad y libertad en la opinión pública y crítica ciudadana a sus acciones.

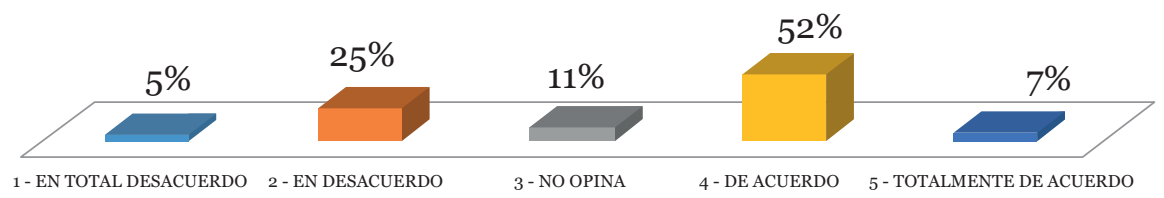

El gobierno municipal es muy respetuoso de la libertad de opinión según el 59\% por ciento de la población objeto de investigación, se mantiene un porcentaje del 31\% de los que no están de acuerdo con esta afirmación. Los encuestados creen que el gobierno municipal a pesar de no compartir a sus críticos no utilizan acciones negativas contra los mismos.

\section{Ítem 3. Los espacios ciudadanos para formular y evaluar políticas públicas}

EN EJERCICIO DE SU AUTORIDAD EL GOBIERNO LOCAL ESTIMULA Y RESPETA: La diversidad y libertad en la opinión pública y crítica ciudadana a sus acciones.

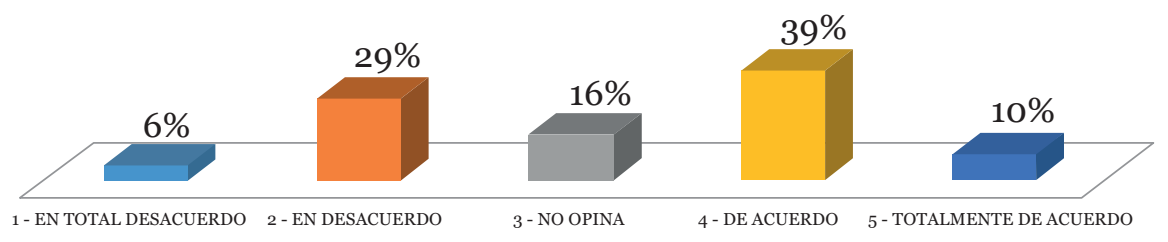

En esta pregunta se nota un porcentaje alto de los ciudadanos que no opinan sobre el tema, un $17 \%$. Un $49 \%$ cree que el gobierno municipal de Valledupar es abierto a lo que diga la ciudadanía en la construcción de las políticas públicas, mientras que el 35\% opina que no está de acuerdo con lo que haciendo la administración municipal con respecto a definir sus políticas públicas junto con la comunidad.

\section{Ítem 4. El acceso a información disponible sobre su gestión}

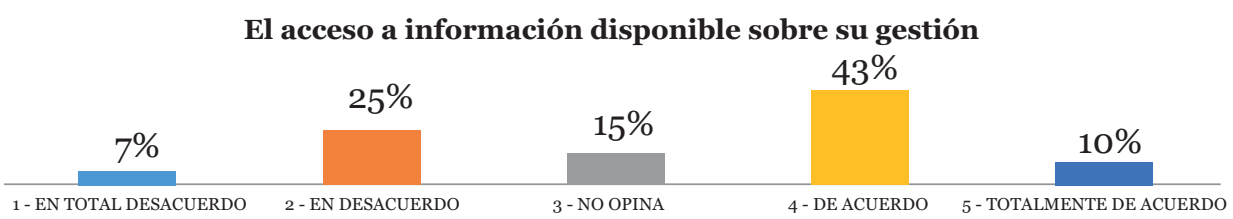


Existe buena información de las actividades de gestión por parte de la administración municipal, así lo manifiesta el $53 \%$ de las opiniones, sin embargo, un porcentaje del $32 \%$ dicen que no, lo que debe generar inquietud de la administración municipal, para que aumente los mecanismos que evidencian las acciones del gobierno local.

\section{Categoría 2: estabilidad política y ausencia de violencia (percepción) Ítem 1. Amenaza política por parte de grupos al margen de la ley}

Amenaza política por parte de grupos al margen de la ley

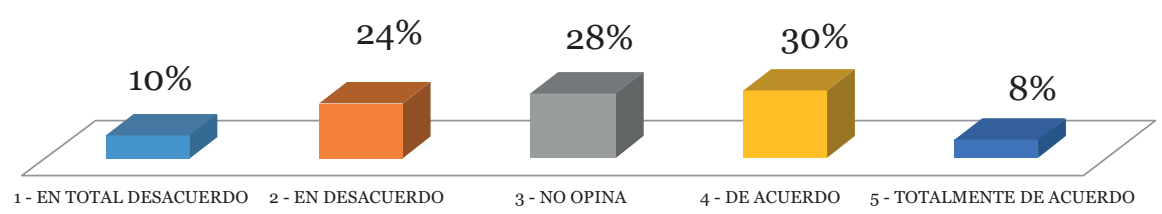

Resalta el porcentaje de los encuestados que no opina sobre este tema, el 28\%, casi la tercera parte de los encuestados, mientras la diferencia entre los que dicen que se siente amenaza política y los que no lo creen llega al $4 \%$, la relación es de $38 \%$ versus $34 \%$ de los primeros con respecto de los segundos, la mayoría se siente amenazada.

Ítem 2. Que el gobierno aplica políticas para minimizar la acción de grupos al margen de la ley

EN EJERCICIO DE SU AUTORIDAD EL GOBIERNO LOCAL ESTIMULA Y RESPETA: Que el gobierno aplica políticas para minimizar la acción de grupos al margen de la ley

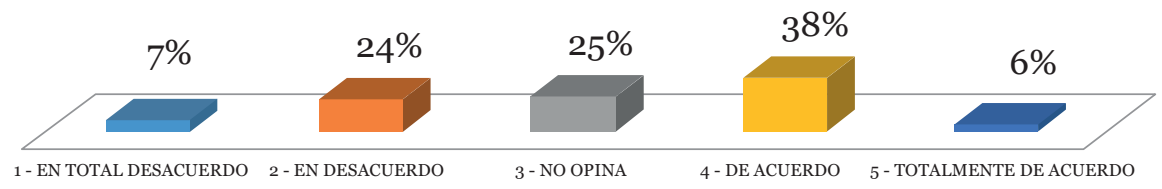

Llama poderosamente la atención que la suma de los que no opinan, nuevamente alta, más lo que creen que la administración municipal no está realizando políticas para minimizar las acciones violentas llegan al 56\%; esta cifra puede ser discutida ya que el hecho de no opinar puede ser por temor o por indiferencia. Por otro lado el $44 \%$ percibe que el gobierno si está trabajando en este tema. 
Ítem 3. Que la financiación de la campaña con que fue elegido el mandatario y el concejo municipal les da legitimidad e independencia como gobernantes

Que la financiación de la campaña con que fue elegido el mandatario y el concejo municipal les da legitimidad e independencia como gobernantes

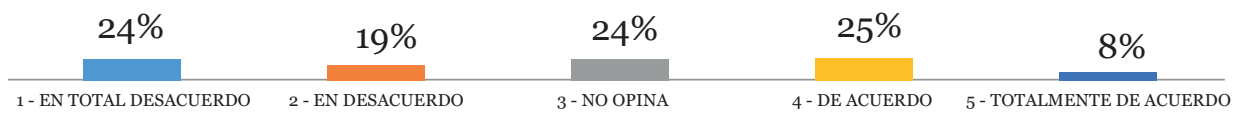

Al comprar las cifras de los que están en total acuerdo contra los que están en total desacuerdo, se observa una distancia notoria, ya que ésta llega al 16\%, entre un $8 \%$ los que creen en la independencia y un $24 \%$ entre los que la ponen en duda. Solamente un $33 \%$ por ciento de los encuestados creen que la administración municipal no está hipotecada a los compromisos políticos adquiridos en campaña electoral.

Ítem 4. Que el grado de protestas cívicas y movimientos en el municipio refleja la eficacia de la administración

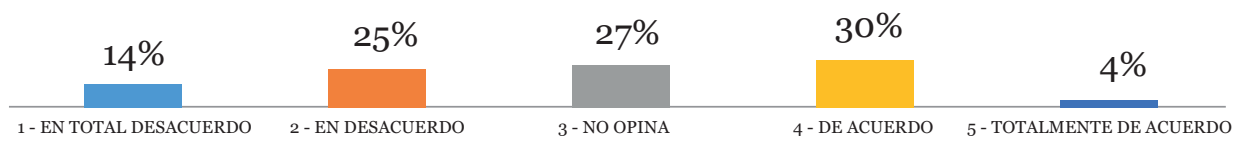

Un 35\% de los sujetos de estudio, piensa que los habitantes del municipio de Valledupar no protesta porque está de acuerdo con la forma como el gobierno municipal ejecutan su gobierno. El 39\%, la mayoría opina que el municipio no es eficaz en su ejercicio, e igualmente se destaca el gran porcentaje de los que no opinan con un 27\%. La ciudadanía es reacia al opi-nar sobre aspectos que pueden ser negativos para la administración.

Categoría 3: eficacia de la acción gubernativa (ejercicio de cumplimiento municipal)

Ítem 1. Resultados eficaces en el corto y mediano plazo en la implementación de políticas públicas

Resultados eficaces en el corto y mediano plazo en la implementación de políticas públicas

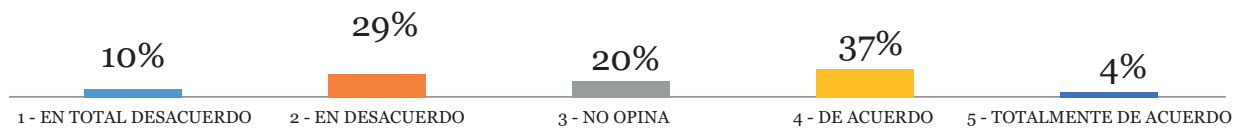


Existe un empate técnico entre los encuestados que apoyan la eficacia en el corto plazo del gobierno municipal y de los que opinan lo contrario, la relación es de $41 \%$ de apoyo contra $39 \%$ en desacuerdo. La opinión está repartida, en el ejercicio del tiempo de la actual administración, a casi dos (2) años de actuación administrativa, la comunidad de Valledupar no ve eficacia en la implementación de políticas públicas, es decir, no está resolviendo los problemas que aquejan a la comunidad.

\section{Ítem 2. Sus propuestas y compromisos de campaña}

Resultados eficaces en el corto y mediano plazo en la implementación de políticas públicas

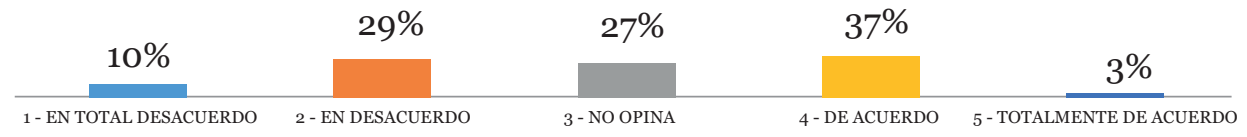

Solamente el 34\% de los encuestados cree que la administración municipal ha cumplido con sus compromisos de campaña, ya en la mitad del periodo de gobierno. El 39\% opina que el gobernante local no ha sido consecuente con sus promesas. Se destaca que técnicamente los que no opinan tiene el mismo porcentaje que los que están de acuerdo y en desacuerdo con un significativo $27 \%$.

Ítem 3. La prestación de los servicios públicos de calidad y la satisfacción de las necesidades de sus gobernados

La prestación de los servicios públicos de calidad y la satisfacción de las necesidades de sus gobernados

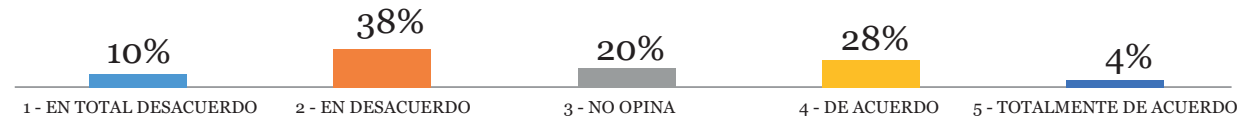

Es categórico el resultado de este ítem; el 49\% de la ciudadanía vallenata, no están satisfechos con los servicios públicos que está prestando el municipio, o con las acciones de gobierno para satisfacer las necesidades de la comunidad. Los que no opinan llegan al 20\%, manteniéndose el alto índice de los encuestados que no se atreven a opinar sobre las acciones del gobierno municipal. Un 32\% les gusta la forma como el gobierno municipal ejecuta acciones para la prestación de servicios públicos e igualmente para solucionar sus necesidades insatisfechas.

Ítem 4. La relación entre la utilización del gasto público y el efecto del mismo en obras es notoria

La relación entre la utilización del gasto público y el efecto del mismo en obras es notoria

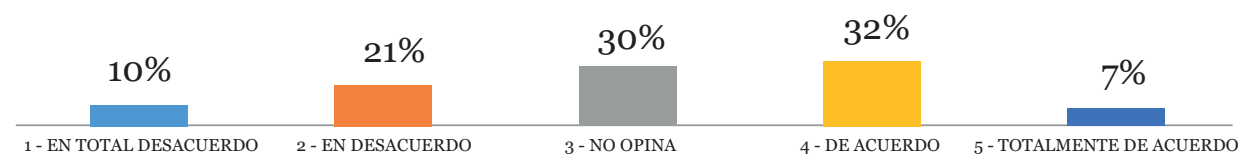


Se desconoce cuáles son las motivaciones de los sujetos encuestados para no opinar, pero en este ítem un 30\% es realmente alarmante. Que motiva a los vallenatos a no opinar sobre el gasto público del gobierno local? Una mayoría exigua del 39\%, con respecto a la muestra total de esta investigación, cree que el gasto público municipal se ve reflejado en el avance de las obras, mientras que el 31\% piensa lo opuesto, no se ve reflejada la inversión en el progreso de las obras que se están ejecutando.

\section{Categoría 4: calidad regulatoria (fomento de normas regulatorias municipales) Ítem 1. Medidas de fomento y dinamización del aparato productivo y mejora de la competitividad}

Medidas de fomento y dinamización del aparato productivo y mejora de la competitividad

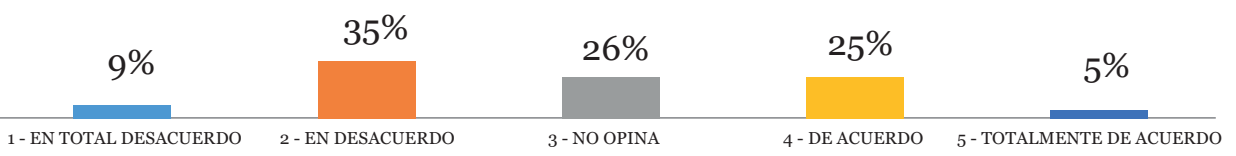

Frente al trabajo desarrollado por la administración municipal para mejorar la productividad y la competitividad del aparato productivo, es significativo que el $44 \%$ de los encuestados opinen que el gobierno del municipio de Valledupar, no ha realizado acciones regulatorias que coadyuven en el fortalecimiento del aparato productivo; la diferencia es de un 14\% frente a los que con un $30 \%$ opinan que si las ha realizado.

\section{Ítem 2. Crecimiento económico, generación de empleo y bienestar social}

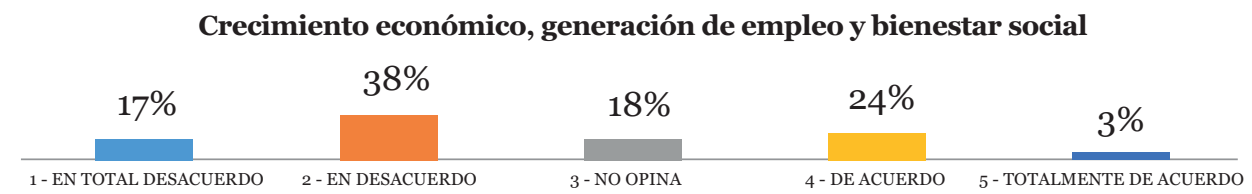

Este indicador respecto de la encuesta realizada en el municipio de Valledupar evidencia que los sujetos de investigación, con un porcentaje muy alto $56 \%$ respecto al tamaño de la muestra, creen que la administración municipal no ha realizado, hasta el momento, acciones que auspicien el crecimiento económico, el empleo y el bienestar social, se percibe el descontento de la comunidad vallenata, toda vez que los que respaldan a las acciones del gobierno municipal solo llegan al 27\%. La distancia entre los que están totalmente de acuerdo y en desacuerdo es de $14 \%$ a favor de los segundos. 


\section{Ítem 3. El fomento y/o su desarrollo y consolidación de las Mipymes}

El fomento y/o su desarrollo y consolidación de las Mipymes

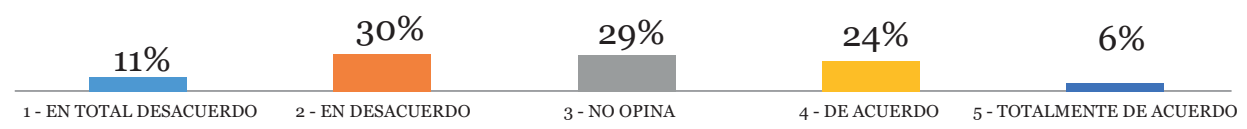

Se mantienen las cifras en este indicador respecto del descontento que tiene la comunidad vallenata con la administración municipal. Mientras el porcentaje de los que sienten que no apoya a los pequeños empresarios es del 41\%, solamente el 29\% opinan que si los apoya. Se resalta que el porcentaje de los que no opinan es superior que los que apoyan al gobernante local.

\section{Categoría 5: imperio de la ley (reflejo del respeto de la ley en el municipio) Ítem 1. El trato digno y reconocimiento a la dignidad humana}

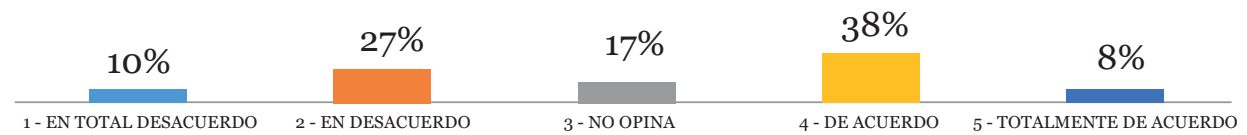

En cuanto al respeto de la administración municipal por la dignidad de los habitantes del municipio, el $46 \%$ cree si lo tiene, se percibe al gobernante como respetuoso de las personas. Sin embargo, es importante el porcentaje de los que creen que no, ya que su porcentaje llega al 37\%, que se puede considerar una cifra alta por tratarse de un tema de derechos fundamentales.

\section{Ítem 2. El modo de proceder de los agentes del orden y servidores públicos}

El modo de proceder de los agentes del orden y servidores públicos

\begin{tabular}{ccrrr}
$14 \%$ & $37 \%$ & $19 \%$ & $25 \%$ & $5 \%$ \\
\hline 1 - EN TOTAL DESACUERDO & 2 - EN DESACUERDO & 3 - NO OPINA & 4- DE ACUERDO & 5- TOTALMENTE DE ACUERDO
\end{tabular}

El 51\% de los encuestados, perciben que el proceder de los agentes del orden y de los servidores públicos no es aceptable; esta cifra es bastante alta, pues, su diferencia con los que creen que si es adecuada, sólo llega al 30\%. 


\section{Ítem 3. El número de delitos y homicidios está dentro de los límites estadís- ticos aceptados}

El número de delitos y homicidios está dentro de los límites estadísticos aceptados

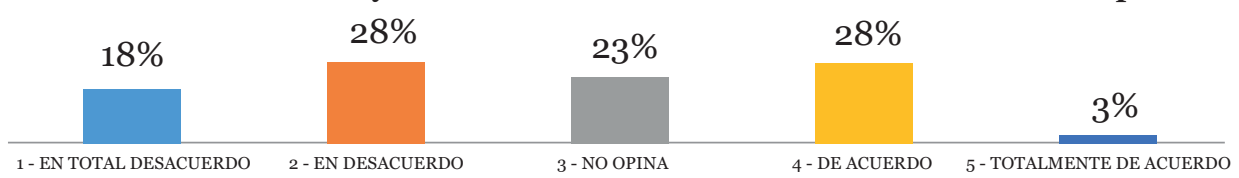

El $46 \%$ de los pobladores de Valledupar piensan que es muy alto el porcentaje de delitos y homicidios, nuevamente este guarismo es notablemente alto, comparado con los que opinan que es normal lo que sucede en el municipio: el 31\%. La diferencia entre estas dos opiniones se muestra claramente respecto de los extremos que opinan en favor y en contra del gobierno municipal, se cuantifica en un $15 \%$, se percibe mucha inseguridad.

\section{Ítem 4. La moralidad y la ecuanimidad en que se fundamentan las normas locales}

La moralidad y la ecuanimidad en que se fundamentan las normas locales

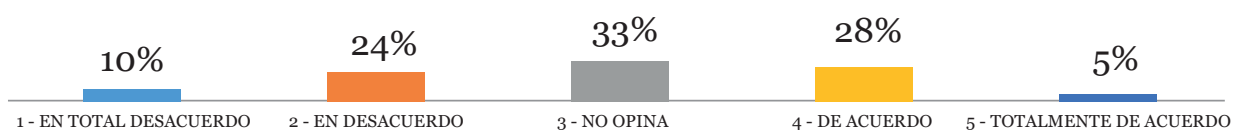

Este indicador es muy particular, se observa una simetría entre los que no opinan en cuanto a la temática, los que creen que hay un gobierno municipal que respeta la moral y que no se deja llevar por la pasión, y los que consideran que por el contrario n la administración municipal no está obrando con rectitud, imparcialidad y justicia. Un 34\% tienen percepción negativa, un 33\% no opina y un 33\% es positivo. Lo que implica una tendencia de la mayoría en su visión de las actuaciones del gobierno municipal como contrarias a la moral y las buenas costumbres.

\section{Ítem 5. La manera como se ejerce y se aplica la justicia}

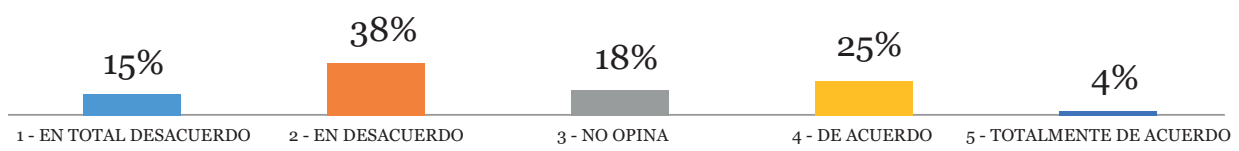

El 52\% de la población encuestada en esta investigación opina que la forma en que se ejerce y se aplica la justicia en el municipio de Valledupar no es adecuada, por lo que no están de acuerdo con el ejercicio normativo desarrollado por el actual gobierno municipal en este tópico. La información muestra que sólo el $4 \%$ de la población apoya en forma total las acciones 
de la administración municipal, porcentaje que no alcanza a superar el margen de error, por lo que se puede inferir que ningún encuestado piensa que lo hecho por el mandatario local hasta ahora es conveniente.

\section{Categoría 6: control y corrupción (control de la autoridad municipal) Ítem 1. La intervención de elites y grupos de poder en las decisiones del gobierno}

La intervención de elites y grupos de poder en las decisiones del gobierno

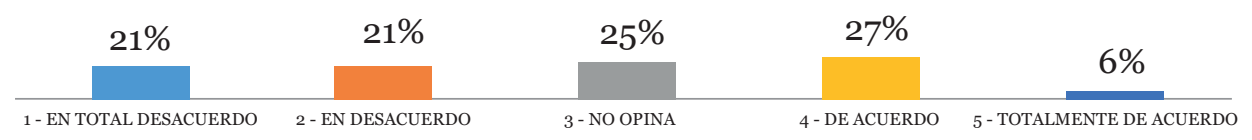

El 33\% apuntala que el gobierno municipal es autónomo en sus actuaciones y decisiones frente a las castas políticas; por el contrario el $42 \%$ piensa que las decisiones de la administración del municipio de Valledupar no controlo la intervención de las elites y grupos de poder por lo que está atado a lo que éstas fijen como prioridades y políticas públicas. Se destaca que existe igualdad técnica entre los que no opinan y los que apoyan la libertad de actuación del gobierno; es una constante que se presenta en este examen que no deja de llamar la atención de los investigadores.

\section{Ítem 2. El manejo transparente de los recursos por parte de los funcionarios}

El manejo transparente de los recursos por parte de los funcionarios

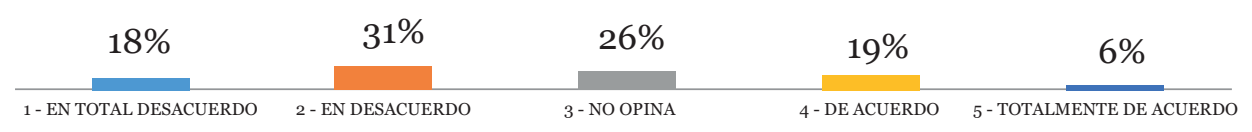

Este indicador es muy inquietante, ya que apenas el 25\% de la población vallenata, percibe un manejo transparente de los recursos por parte de los funcionarios del municipio; un $49 \%$ percibe un inadecuado manejo, es considerable el porcentaje que no comparte la forma como se administran los recursos. Este indicador del municipio de Valledupar, muestra que la mayoría siente que la administración municipal es corrupta.

\section{Ítem 3. Transparencia en la contratación estatal}

Que el asignación de la contratación estatal sea transparente

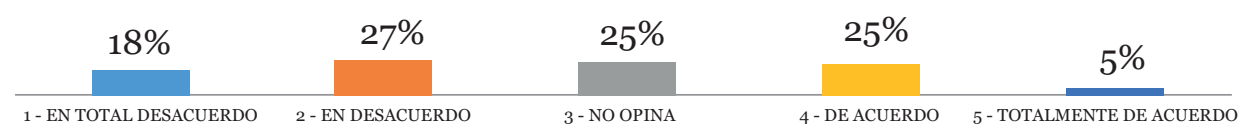

No es transparente la contratación en el municipio de Valledupar, es la dictamen del 45\% de la población encuestada. Un $25 \%$ cree que existe transparencia en la forma como contrata el gobierno municipal, este porcentaje no supera a los que no opinan sobre esto que es de un $25 \%$. 


\section{Ítem 4. La corrupción a través de controles claras}

La corrupción a través de controles claras

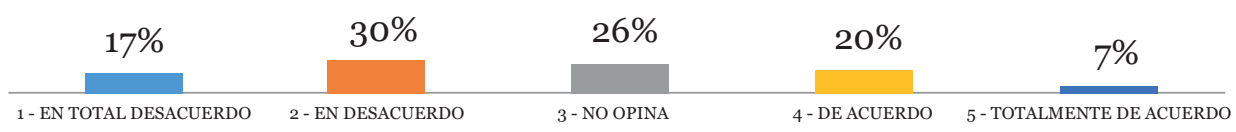

El 21\% por ciento de los sujetos de la investigación creen que el municipio de Valledupar tiene controles claros para enfrentar la corrupción, este guarismo no alcanza a superar a los que no opinan sobre el tema. Mientras que, el alto porcentaje del $47 \%$ percibe a la administración municipal indiferente a los controles claros contra la corrupción, no se percibe esto como prioridad en la actual administración municipal.

\section{Ítem 5. Las prácticas de enriquecimiento ilícito de personas vinculadas a la administración y terceros cercanos}

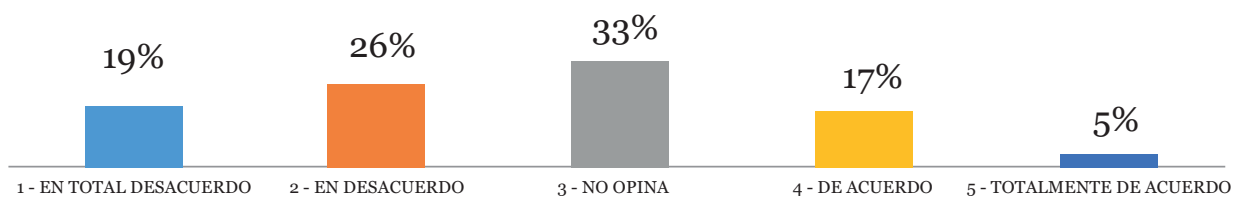

En este interrogante lo llamativo es que el 33\% de los encuestados no quiere opinar sobre el tema. Sólo el $17 \%$ de la población cree que la administración municipal controla el enriquecimiento ilícito de personas pertenecientes a la administración así como la de terceros afectos a la administración del municipio. Es grande el porcentaje de los que piensan que el gobierno municipal de Valledupar está permitiendo que la ilicitud reine en su administración en detrimento del patrimonio público, lo demuestra la cifra del $45 \%$ de los que fueron auscultados en esta investigación.

\section{Categoría 7: gobernabilidad y proceso de paz (acciones del gobierno local) Ítem 1. Ha mostrado interés para que el proceso de paz tenga impacto en el municipio}

Ha mostrado interés para que el proceso de paz tenga impacto en el municipio

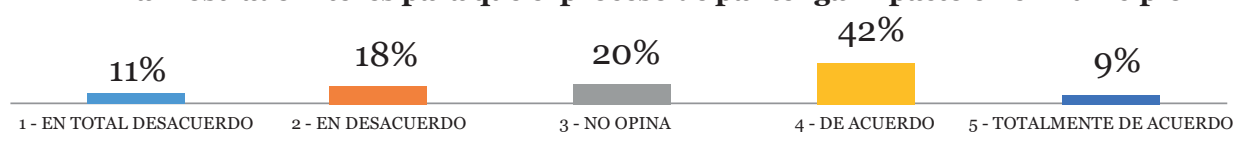

En este indicador es importante el número de personas que cree que al gobierno municipal le interesa que el proceso de paz, firmado por el gobierno nacional y la FARC, tenga impacto en el municipio, lo dice la cifra del $51 \%$. Sólo un $11 \%$ por ciento sienten lo contrario en forma tajante, por lo que se puede inferir un gobierno municipal cercano a la promoción del proceso de paz. 
ANTONIO YESID PEDROZA ESTRADA

DAD Y POSCONFLICTO EN EL MUNICIPIO DE VALLEDUPAR, COLOMBIA

Págs. 129 - 153. DOI: http://dx.doi.org/10.30972/rfce.2324023

\section{Ítem 2. Permite contribuir a generar ambiente de posconflicto en el municipio}

Permite contribuir a generar ambiente de posconflicto en el municipio

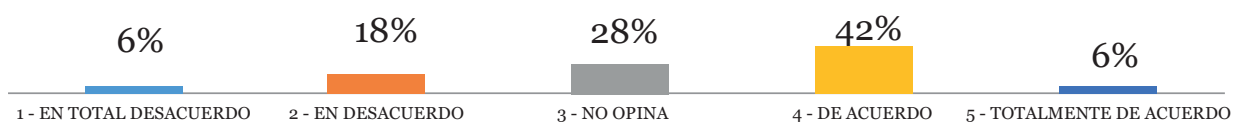

El $48 \%$ por ciento de los encuestados sienten que el gobierno municipal genera un buen ambiente para el posconflicto en el municipio. Es muy bajo el porcentaje de los que opinan que el gobierno municipal no le interesa el posconflicto con un 24\%. A pesar que se mantienen altos los porcentajes de los que no opinan, en general se percibe una administración municipal interesada y con de deseos de apoyar las políticas para enfrentar el posconflicto.

\section{Ítem 3. Propicia espacios ciudadanos para la evaluación de las políticas pú- blicas y programas de gobierno}

Propicia espacios ciudadanos para la evaluación de las políticas públicas y programas de gobierno

\begin{tabular}{ccrrr}
$8 \%$ & $26 \%$ & $26 \%$ & $33 \%$ & $7 \%$ \\
\hline 1- EN TOTAL DESACUERDO & 2- EN DESACUERDO & 3 - NO OPINA & 4- DE ACUERDO & 5- TOTALMENTE DE ACUERDO
\end{tabular}

Se encontró que la comunidad en un 40\% percibe a una administración municipal abierta a las críticas con respecto a las políticas públicas que se desarrollan para enfrentar el posconflicto. Teniendo en cuenta que los que no están de acuerdo con lo afirmado anteriormente llegan a un porcentaje del $34 \%$, se puede establecer una diferencia que esta sólo un (1\%) punto por encima del margen de error, si a esto se le añade la tendencia que se ha mantenido entre los encuestados de no opinar, que para este ítem es alto, un 26\%, se puede inferir una división clara sobre apreciar que el gobierno municipal respecto a propiciar espacios de participación ciudadana con respecto a la temática del posconflicto.

\section{Ítem 4. Ha diseñado políticas orientadas a generar y favorecer un ambiente de posconflicto en la ciudadanía}

Ha diseñado políticas orientadas a generar y favorecer un ambiente de posconflicto en la ciudadanía

\begin{tabular}{crrrr}
$9 \%$ & $23 \%$ & $31 \%$ & $31 \%$ & $6 \%$ \\
\hline 1 - EN TOTAL DESACUERDO & 2 - EN DESACUERDO & 3 - NO OPINA & 4- DE ACUERDO & 5 - TOTALMENTE DE ACUERDO
\end{tabular}

Se observa en este ítem, una constante en esta investigación, el alto indicador de ciudadanos que no quiere opinar sobre la temática, aquí llega al 31\%, porcentaje igual de quienes están de acuerdo en opinar que la administración municipal diseña políticas públicas para favorecer 
un ambiente de posconflicto, con un 31\%, los que están totalmente de acuerdo son el 6\%; mientras que los que están en desacuerdo suman en total 32\%. Teniendo en cuenta el porcentaje de error de la investigación, debemos decir que se presenta un empate entre las dos tendencias.

\section{Ítem 5. Ha demostrado con acciones y programas concretos su compromiso con el proceso del posconflicto}

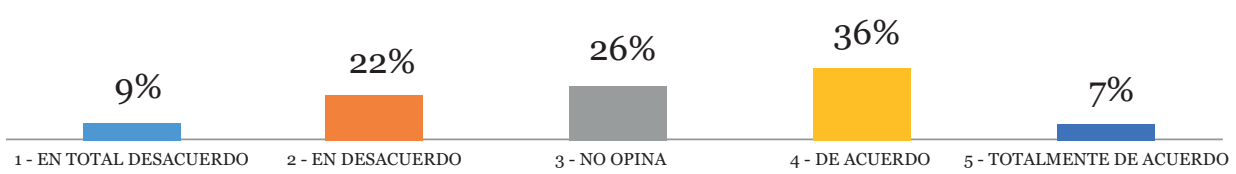

El $43 \%$ cree que este gobierno municipal tiene muestras concretas y fehacientes que lo muestran comprometido con la implementación de acciones que vislumbren éxito del proceso de paz en la etapa de posconflicto. Pero, el $31 \%$ opina lo contrario, y el $26 \%$ no opina.

\section{CONCLUSIONES}

1. En general es alto el porcentaje de los entrevistados que no opinan sobre la administración municipal sobre todo en temas relacionados con el orden público y corrupción, prefieren callar a decir lo que piensan; se puede entender por la situación de violencia vivida en el municipio respecto al tema político que aún tiene eco en la población generando miedo.

2. Se percibe un gobierno respetuoso de la opinión ciudadana crítica, no se le percibe como violento o perseguidor de su oposición.

3. En general, se percibe, contundentemente, una administración municipal corrupta, con malas prácticas de contratación y poca claridad en el manejo de los recursos públicos. Se piensa que el gobierno permite que alguno de sus funcionarios y terceros adeptos al mismo se enriquezcan a expensas del erario público.

4. Se siente que la administración pública no le está ofreciendo servicios públicos adecuados a la población vallenata, ni solucionando sus principales problemáticas.

5. Mucha sensibilidad social en la temática de seguridad y actuar negativo de la fuerza pública, el pueblo vallenato cree que este gobierno no se hace lo necesario para disminuir estas tendencias de delitos y homicidios.

6. Se siente un gobierno municipal atado a las castas políticas sometido a sus compromisos con los grupos de poder, pero que no le ha cumplido, en cambio, a la comunidad respecto a sus promesas de campaña. 
7. Sin embargo, con respecto a las políticas que permitan un desarrollo adecuado del posconflicto se nota que el pueblo vallenato piensa que el gobierno municipal está actuando en consonancia, está abierto a críticas, así como apoyarlo.

8. El pueblo vallenato cree que el gobierno municipal está trabajando por la cultura ciudadana y es respetuoso de la opinión, y que a pesar de no compartir a sus críticos no utiliza acciones negativas contra los mismos.

9. La mayoría del pueblo vallenato se siente amenazado políticamente, explica en parte este indicador que la gente no opina abiertamente cuando se les pregunta por la gestión municipal.

10. La mayor parte de los encuestados cree que la administración municipal está hipotecada a los compromisos políticos adquiridos en campaña electoral.

\section{RESPECTO AL POSCONFLICTO}

1. Es importante el número de personas que cree que al gobierno municipal le interesa que el proceso de paz, firmado por el gobierno nacional y la FARC, tenga impacto en el municipio, lo dice la cifra del $51 \%$.

2. El $48 \%$ por ciento de los encuestados sienten que el gobierno municipal genera un buen ambiente para el posconflicto en el municipio y que propicia espacios de participación ciudadana con respecto a la temática del posconflicto.

\section{RECOMENDACIONES}

1. La administración municipal debe mantener comunicación abierta, sincera, constante con la ciudadanía reforzando las estrategias de comunicación construyendo las bases de un gobierno abierto, receptivo, rindiendo cuentas de manera constante utilizando los medios tecnológicos y haciendo las rendiciones de cuenta por lo menos dos (2) veces al año, tal y como lo estipula la Ley colombiana.

2. El alcalde debe implementar mecanismos de seguimiento y control ético a su equipo directivo, enviando un mensaje a la comunidad que él como cabeza del gobierno municipal ejerce liderazgo siendo un referente de la administración trabajando contra conductas y fenómenos de corrupción; se le recomienda contratar un equipo auditor externo que le informe sobre posibles fallas de su equipo, ponerlo en conocimiento de la comunidad y ejercer medidas drásticas contra acciones corruptas. 
3. El Alcalde debería cambiar su estrategias comunicacionales actuales a través de las cuales se le rinde culto a su imagen, su familia y su iglesia, desviando la atención de las acciones, políticas públicas y proyectos desarrollados por la administración en beneficio de la sociedad vallenata; darle dignidad y honorabilidad al cargo de alcalde suprimiendo lo coloquial y folclórico al anunciarlo sólo como "tuto", siendo lo ideal utilizar su nombre de pila mostrando respeto a la comunidad.

4. Demostrar con hechos y acciones contundentes que el gobierno municipal obedece a un modelo instaurado por el Alcalde y no por los gamonales políticos tradicionales que gobiernan tras bambalinas dándole una imagen de títere sin criterio y capacidad de decidir lo conveniente para el municipio, su comunidad y no para los contratistas impuestos por los grupos de poder imperantes en el municipio.

\section{REFERENCIAS BIBLIOGRÁFICAS}

BANCO MUNDIAL (BM). (2017). Indicadores de gobernabilidad del Banco Mundial. México: FCE.

BANCO MUNDIAL (BM). (2017). Informe sobre el desarrollo mundial 2007. Desarrollo y la próxima generación: Informe sobre el desarrollo mundial: El desarrollo y la nueva generación. 2007. Disponible en: http://documentos.bancomundial.org/curated/es/home Recuperado 13/04/2017.

Berensztein, S. (2007). Indicadores mundiales de gobernabilidad. Documento de trabajo. Buenos Aires: Instituto para el desarrollo empresarial de la Argentina, IDEA.

Bryman, A. (1992). Carisma AndLeadership in Organizations. London: Sage Publications.

Calderón, J. (2017). Escritos I. Artículos y discursos. Valledupar: Cámara de Comercio.

Club de Roma. (1996). La capacidad de gobernar: Informe al Club de Roma/de Yehezkel Dror; trad. de Carmen Aguilar. México: FCE.

Echebarría, K. (2004). Objetivar la gobernanza: funciones y metodología. Una aproximación a la objetivación del análisis institucional a través de indicadores de gobernabilidad. Madrid: IX Congreso internacional del CLAD sobre la reforma del Estado, Nov. 2-5.

Fossatti, P. (2013). Perfil docente e produção de sentido. Canoas: Editora Unilasalle. 
Fundación este País. (2017). Indicadores de gobernabilidad del Banco Mundial. México: FEP. Escuela Superior de Administración Pública, ESAP.(2015). Fundamentos de Gobernabilidad democrática. Valledupar: ESAP.

Hernández, R. C. (2016). Indicadores de gobernabilidad. México: Firmas. Disponible en: http://www.milenio.com/firmas/ricardo_cisneros_hernandez/Indicadores-gobernabilidad_18_778902118.html. 18/05/2017

Hernández, R., Fernández \& Batista. (2016). Metodología de la investigación. México: Editorial McGraw-Hill.

Hollander, E.P. (1978). Leadership Dynamics: A Pratical Guide To Effective Relationsships. New York: The Free Press.

Hurtado, J. (2010). El proyecto de investigación. Comprensión holística de la metodología y la investigación. Bogotá: Quirón ediciones.

Kauffmann, D. \& Kraay, A. (2008). Gobernabilidad y crecimiento en el largo plazo: Evidencia para Latinoamérica y el Mundo. Washington: Banco Mundial.

Martínez, U. S., Giraldo, O. \& Paniagua, R. (2014). Gobernabilidad, Participación y cultura de paz. Valledupar: Agencia de Cooperación Alemana - Programa de Desarrollo y Paz del Cesar, PDPC.

Oficina del Alto Comisionado para la Paz. (2017). Mesa de negociaciones de La Habana. Acuerdo final para la terminación del conflicto y la construcción de una paz estable y duradera. Bogotá: Oficina del Alto Comisionado para la Paz.

Programa de las Naciones Unidas para el Desarrollo, PNUD. Indicadores de gobernabilidad. Guía del usuario. Oslo: Centro de Gobernabilidad de Oslo. (s.f.)

Programa de las Naciones Unidas para el Desarrollo, PNUD. (2008). Global Integrity, Guía del usuario para medir la corrupción. Disponible en: http://ww38.gaportal.org/sites/default/files/users_guide_measuring_corruption_spanish.pdf Recuperado el 01/o6/ 2017.

Centro de gobernabilidad de Oslo. (2014). Guía del usuario para medir la gobernabilidad local. Oslo: Centro de Gobernabilidad de Oslo. Disponible en: www.gaportal.org/sites/default/ files/usersguide_localgov_ogco9_es_o.pdf. Recuperado el 10/05/2017. 
Universidad del Rosario. (2017). Experimentos de reconciliación política en Colombia. Disponible en:https://www.urosario.edu.co/jurisprudencia/jurisprudencia-reconciliacion/ur/Postconflicto/ Recuperado: 03/05/2017.

http://www.valledupar-cesar.gov.co/Paginas/default.aspx

\section{CURRICULUM VITAE}

\section{Antonio Yesid Pedroza Estrada}

Doctor en Ciencias Políticas. Docente investigador de la Escuela Superior de Administración Pública (ESAP) y de la Universidad Popular del Cesar (UPC), Colombia.

yesithpedroza@hotmail.com 